\title{
High-sensitivity gamma-glutamyltransferase fraction pattern in alcohol addicts and abstainers
}

\author{
Maria Franzini ${ }^{\mathrm{a}, \mathrm{c}, *}$, Irene Fornaciari ${ }^{\mathrm{a}}$, Tiziana Vico ${ }^{\mathrm{b}}$, Marco Moncini $^{\mathrm{b}}$, Valerio Cellesi $^{\mathrm{b}}$, \\ Milo Meini ${ }^{\mathrm{b}}$, Michele Emdin ${ }^{\mathrm{c}}$, Aldo Paolicchi ${ }^{\mathrm{c}, \mathrm{d}}$ \\ a Scuola Superiore Sant'Anna, Pz. Martiri della Libertà 33, 56127 Pisa, Italy

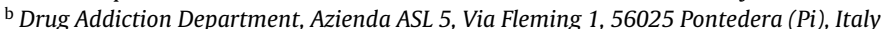 \\ ' Division of Cardiovascular Medicine, Fondazione G. Monasterio CNR - Regione Toscana, Via Giuseppe Moruzzi 1, 56124 Pisa, Italy \\ d Department of Experimental Pathology, University of Pisa, Via Roma 55, 56126 Pisa, Italy
}

\section{A R T I C L E I N F O}

\section{Article history:}

Received 22 December 2011

Received in revised form 12 April 2012

Accepted 4 June 2012

Available online $\mathrm{xxx}$

\section{Keywords:}

Alcohol

Biomarker

Gamma-glutamyltransferase fractions

Gel filtration chromatography

\begin{abstract}
A B S T R A C T
Background: Four fractions of gamma-glutamyltransferase (GGT) with different molecular weight (b-, m-, $\mathrm{s}-$, and $\mathrm{f}-\mathrm{GGT}$ ) are present in human plasma. Differential GGT fraction pattern is found in non-alcoholic liver disease (NAFLD) and chronic viral hepatitis, characterized by normal or decreased b-GGT/s-GGT (b/s) ratio, respectively.

Methods: Chromatographic fractional GGT analysis was performed on plasma obtained from 51 subjects: 27 alcoholics (mean (SD), age 45 (9) years; 23 males; 14 positive for viral infection), 24 abstinents from at least 1 month (43 (12) years; 20 males; 6 positive for viral infection). Twenty-seven blood donors matched for age and gender (44 (9) years; 23 males) were selected as controls.

Results: All fractions were significantly increased in alcoholics $(P<0.001)$, s-GGT showing the largest increase, while only m-GGT and s-GGT were elevated in abstainers $(P<0.01)$, in comparison with controls. $\mathrm{The} \mathrm{b} / \mathrm{s}$ ratio was significantly lower in both alcoholics and abstainers than in controls (median (25th-75th perc.): 0.10 (0.07-0.15), $0.16(0.10-0.24), 0.35(0.29-0.53)$, respectively, $P<0.001)$. Viral infection did not significantly changes absolute values of individual GGT fractions in alcoholics, but the $\mathrm{b} / \mathrm{s}$ ratio was significantly lower in virus positive than in virus negative subjects $(0.08(0.05-0.12), 0.14(0.09-0.20)$, respectively, $P<0.01$ ).

Conclusions: The fraction pattern analysis might increase the specificity of GGT as biomarker of alcohol abuse, especially concerning the differential diagnosis between alcoholism and NAFLD, a common cause of elevated GGT level in the general population.
\end{abstract}

(C) 2012 Elsevier Ireland Ltd. All rights reserved.

\section{Introduction}

Serum gamma-glutamyltransferase (GGT) is a well-recognized sensitive biomarker of alcohol abuse (Rosalki and Rau, 1972; Das et al., 2008). GGT specificity and diagnostic accuracy for alcoholism are reduced by the fact that serum GGT elevated levels are encountered in various physiological and pathological setting including a wide array of hepatobiliary disorders (Dhanya and Vasudevan, 2008). In particular, a common cause of serum GGT increase is the non-alcoholic fatty liver disease (NAFLD), that affects a growing proportion of the population (Bedogni et al., 2005; Tahan et al.,

\footnotetext{
* Corresponding author at: Scuola Superiore Sant'Anna, c/o Fondazione Toscana G. Monasterio, Via G. Moruzzi 1, 56124 Pisa, Italy. Tel.: +39 0503153309 ; fax: +39050 3152166 .

E-mail addresses: m.franzini@sssup.it, franzinimaria@gmail.com (M. Franzini).
}

2008), thus the possibility of discriminating between the two diseases would help the diagnosis of both conditions.

Total GGT activity corresponds to several distinct GGTcontaining molecular complexes, with different physico-chemical properties (Huseby, 1982), pathophysiological and clinical correlations (Nemesanszky and Lott, 1985). Recently, a high sensitivity clinical laboratory method has become available, that allows, in human plasma, the simultaneous detection of four different fractions of GGT, namely b-GGT, m-GGT, s-GGT, and f-GGT, having molecular weight ranging from 2000 to $70 \mathrm{kDa}$ (Franzini et al., 2008a). While f-GGT is the most abundant GGT fraction in healthy individuals (Franzini et al., 2008b), the other fractions are responsible for GGT increase in diseased ones. Recently, we found different GGT fraction patterns characterizing either NAFLD, or chronic viral hepatitis $\mathrm{C}(\mathrm{CHC})$ : the former was characterized by an increase in all fractions without changing in the b-GGT/s-GGT (b/s) ratio, and the later by a prominent increase in s-GGT together with a modest 
Table 1

Characteristics of controls, alcohol addicts and abstainers.

\begin{tabular}{|c|c|c|c|c|c|c|}
\hline & Controls $(n=27)$ & Alcoholics $(n=27)$ & Abstainers $(n=24)$ & $\begin{array}{l}P \\
\text { Controls vs. } \\
\text { alcoholics }\end{array}$ & $\begin{array}{l}P \\
\text { Controls vs. } \\
\text { abstainers }\end{array}$ & $\begin{array}{l}P \\
\text { Alcoholics vs. } \\
\text { abstainers }\end{array}$ \\
\hline Males, $n$ & 23 & 23 & 20 & & & \\
\hline Age, years & $44.0(35.0-48.0)$ & $44.0(36.0-48.0)$ & $39.0(35.5-53.0)$ & n.s. & n.s. & n.s. \\
\hline BMI, $\mathrm{kg} / \mathrm{m}^{2}$ & $24.8(22.9-27.0)$ & $23.5(21.6-27.3)$ & $24.3(22.0-26.1)$ & n.s. & n.s. & n.s. \\
\hline AST, $\mathrm{U} / \mathrm{L}$ & $20.0(17.0-23.0)$ & $58.0(31.0-96.0)$ & $22.5(18.0-33.0)$ & $<0.001$ & n.s. & $<0.001$ \\
\hline ALT, U/L & $24.0(15.0-26.0)$ & $57.0(25.0-95.0)$ & $29.0(15.0-41.5)$ & $<0.01$ & n.s. & $<0.05$ \\
\hline $\mathrm{MCV}, \mathrm{fL}$ & $85.9(83.3-87.5)$ & $95.1(89.2-102.3)$ & $93.1(91.0-95.3)$ & $<0.001$ & $<0.001$ & n.s. \\
\hline Bilirubin, mg/dL & $0.80(0.60-0.90)$ & $0.76(0.45-1.11)$ & $0.44(0.32-0.81)$ & n.s. & n.s. & n.s. \\
\hline Total GGT, U/La & $23.9(15.1-32.0)$ & $133.0(50.1-287.0)$ & $37.5(19.4-84.8)$ & $<0.001$ & $<0.05$ & $<0.001$ \\
\hline b-GGT, U/L $\mathrm{L}^{\mathrm{a}}$ & $2.4(1.4-4.5)$ & $7.5(3.2-21.0)$ & $2.8(1.3-7.6)$ & $<0.001$ & n.s. & $<0.01$ \\
\hline $\mathrm{m}-\mathrm{GGT}, \mathrm{U} / \mathrm{L}^{\mathrm{a}}$ & $1.0(0.5-1.5)$ & $5.2(2.8-19.0)$ & $2.1(0.8-4.5)$ & $<0.001$ & $<0.01$ & $<0.001$ \\
\hline $\mathrm{s}-\mathrm{GGT}, \mathrm{U} / \mathrm{L}^{\mathrm{a}}$ & $7.3(3.6-12.4)$ & $95.7(30.1-207.8)$ & $15.3(8.1-58.5)$ & $<0.001$ & $<0.01$ & $<0.001$ \\
\hline f-GGT, U/L & $13.7(9.9-16.9)$ & $22.7(15.4-26.7)$ & $14.4(9.0-18.8)$ & $<0.001$ & n.s. & $<0.001$ \\
\hline $\mathrm{b} / \mathrm{s}$ ratio ${ }^{\mathrm{a}}$ & $0.35(0.29-0.53)$ & $0.10(0.07-0.15)$ & $0.16(0.10-0.24)$ & $<0.001$ & $<0.001$ & $<0.05$ \\
\hline
\end{tabular}

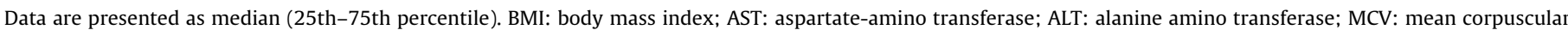
volume; GGT: gamma-glutamyltransferase. Statistical analysis: 1-way ANOVA followed by Tukey's multiple comparison test. n.s.: not significant.

a Statistical analysis performed on ln-transformed data.

increase in other fractions and decrease of the $\mathrm{b} / \mathrm{s}$ ratio (Franzini et al., 2011).

Since steatosis is a main feature of hepatocellular damage occurring early in the course of alcoholic liver disease (ALD; Basra and Anand, 2011), we aimed to check whether the GGT fraction pattern associated with ALD could mimic those found in NAFLD (Franzini et al., 2011), in a preliminary series of alcohol addicts. This would permit to design large-scale studies concerning the diagnostic value of GGT fraction analysis in the setting of alcohol abuse.

\section{Methods}

\subsection{Subjects}

Fasting blood samples were obtained from 51 subjects with positive history of alcohol dependence according to the ICD-9-CM Diagnosis Code 303.9 (mean (SD; range): age 43.6 (10.6; 26-69) years; 45 males and 6 females; abuse duration 4.6 (range 1.3-20) years). All these subjects were enrolled in the study at the Alcoholism Center of the Drug Addiction Department of the Local Health Service (Pisa, Italy).

Alcohol dependence and consumption were estimated by physicians of the Alcoholism Center based upon the information collected from the patient as well as from his/her relatives during the interview. The alcohol content of one standard drink in Italy is assumed to be $12 \mathrm{~g}$ of pure alcohol (web site: http://icap.org/PolicyIssues/DrinkingGuidelines). According to WHO recommendations we set the limit of hazardous alcohol consumption to $>45 \mathrm{~g} /$ day for men and $>30 \mathrm{~g}$ /day for women (Schellenberg et al., 2005).

Twenty-four subjects were abstinent for at least 1 month (age $43.3(12.1 ; 26-69)$ years; 20 men; abstinence time: 2.7 (3.4) months): these subjects will be referred as "abstainers". Six abstainers were either hepatitis C virus (HCV) or hepatitis B virus (HBV) antibodies positive. Abnormalities in both alanine (ALT) and aspartate-amino transferase (AST) have been detected in 3 abstainers. The other 3 abstainers showed ALT and/or AST elevation.

Twenty-seven subjects were currently alcohol dependent (age 44.9 (9.2; 30-65) years; 23 men; alcohol consumption: 209 (192; 100-810) g/day). Fourteen subjects (age 40.1 (6.5; 30-50) years; 12 men; alcohol consumption 162 (78; 80-290) g/day) were HCV or HBV antibodies positive with both AST and ALT elevated levels, 10 among them were proved positive for HCV-RNA test or HBV surface antigen (HBsAg). The remaining 13 subjects (age 48.0 (10.1; 33-65) years; 11 men; alcohol consumption $250(254 ; 100-810) \mathrm{g} /$ day $)$ showed negative virology analysis, among them, 7 showed AST and ALT elevation.

Twenty-seven blood donors matched for age and gender have been selected as controls (age 43.7 (9.3; 27-64) years; 23 men; alcohol consumption $\leq 20$ g/day).

The Institutional Ethics Committee approved the study and all subjects gave informed consent.

\subsection{Laboratory analyses}

Fasting blood samples, collected in ethylenediamine-tetra-acetic acid (EDTA), were obtained from all subjects. AST, ALT, mean corpuscular volume (MCV), total bilirubin, serum glucose, total cholesterol, HDL cholesterol, LDL cholesterol, triglycerides and creatinine clearance (eGFR) were assayed within $3 \mathrm{~h}$ according to the standard clinical laboratory procedures by automated analyzers (Beckman Synchron CX 9-PRO analyzer, Abbott Cell-Dyn Saphire for blood cell count). LDL cholesterol and eGFR were calculated using the Friedewald and the Cockcroft-Gault formula, respectively.

\subsection{Fractional GGT analysis}

Aliquots of plasma EDTA samples were stored at $-20^{\circ} \mathrm{C}$ and used within three months for fractional GGT analysis. Analysis of total and fractional GGT was performed, as previously described (Franzini et al., 2008a,b), using a fast protein liquid chromatography system (AKTA purifier; GE Healthcare Europe, Milan, Italy) equipped with a gel-filtration column (Superose 6 HR 10/300 GL; GE Healthcare Europe) and a fluorescence detector (Jasco FP-2020; Jasco Europe, Lecco, Italy) The enzymatic activity was quantified by post-column injection of the fluorescent substrate for GGT, gamma-glutamyl-7-amido-4-methylcoumarin (gGluAMC). Enzymatic reaction, in the presence of gGluAMC $0.030 \mathrm{mmol} / \mathrm{L}$ and glycylglycine $4.5 \mathrm{mmol} / \mathrm{L}$, proceeded for $4.5 \mathrm{~min}$ in a reaction coil (PFA, $2.6 \mathrm{~mL}$ ) kept at the $37^{\circ} \mathrm{C}$ in a water bath. The fluorescence detector operating at excitation/emission wavelengths of $380 / 440 \mathrm{~nm}$ detected the AMC signal. Area under curve chromatogram curve is proportional to GGT activity, which was quantified as previously described (Franzini et al., 2008b).

\subsection{Statistical analysis}

Statistical analysis was conducted by one-way ANOVA analysis followed by Tukey's multiple comparison test or Student's $t$ test. Total, b-, m- and s-GGT, as well as $b / s$ ratio and triglyceride values were ln-transformed to reduce the distribution skewness.

\section{Results}

As expected, significant elevation of aspartate-amino transferase (AST, $P<0.001$ ), alanine-amino transferase (ALT, $P<0.01$ ), and mean corpuscular volume (MCV, $P<0.001)$ was detected in alcohol addicts as compared to controls. Abstainers showed an increase in MCV values only $(P<0.001$; Table 1$)$.

Although total GGT values were significantly increased in alcohol addicts and abstainers as compared to controls $(P<0.001$ and $<0.05$, respectively), while all GGT fractions were significantly higher in alcoholics $(P<0.001)$, only $\mathrm{m}-\mathrm{GGT}$ and s-GGT remained significantly elevated in abstainers $(P<0.01$; Table 1$)$. In alcohol addicts, a 13.1-fold increase value in s-GGT was detected, together with a significant but more modest elevation in all other fractions (f-GGT: 1.7-fold; b-GGT: 3.1-fold; m-GGT: 5.2-fold). In abstainers, as stated above, the values of both m-GGT and s-GGT fractions remained significantly higher than in controls, but at a much lower extent (2.1-fold for both). Thus the b/s ratio remained lower in both groups $(P<0.001)$, than in controls, with a higher $\mathrm{b} / \mathrm{s}$ ratio in abstainers than in addicts $(P<0.05$; Table 1$)$.

Among addicts, no significant differences as concerns each GGT fraction were found between the HCV/HBV positive or $(n=14)$ or negative $(n=13)$ subjects, except for the $\mathrm{b} / \mathrm{s}$ ratio, that was 
Table 2

Total and fractional GGT activity (U/L) in HCV/HBV positive or negative alcohol addicts and abstainers.

\begin{tabular}{|c|c|c|c|}
\hline Alcoholics & HCV/HBV negative $(n=13)$ & HCV/HBV positive $(n=14)$ & $P$ \\
\hline Total GGTa & $168.0(45.7-695.4)$ & $129.0(73.2-274.5)$ & n.s. \\
\hline b-GGT ${ }^{a}$ & $9.0(3.0-60.0)$ & $5.6(3.4-14.4)$ & n.s. \\
\hline $\mathrm{m}-\mathrm{GGT}^{\mathrm{a}}$ & $8.4(2.5-32.2)$ & $4.5(2.9-15.3)$ & n.s. \\
\hline s-GGT ${ }^{a}$ & $112.9(24.6-502.1)$ & $95.6(48.8-205.3)$ & n.s. \\
\hline f-GGT & $22.7(14.0-55.3)$ & $22.7(18.0-25.5)$ & n.s. \\
\hline $\mathrm{b} / \mathrm{s}$ ratio ${ }^{\mathrm{a}}$ & $0.14(0.09-0.20)$ & $0.08(0.05-0.12)$ & 0.0045 \\
\hline Abstainers & HCV/HBV negative $(n=18)$ & HCV/HBV positive $(n=6)$ & $P$ \\
\hline Total GGT & $37.5(18.0-261.8)$ & $47.1(25.0-130.3)$ & n.s. \\
\hline b-GGT ${ }^{a}$ & $2.7(1.1-25.6)$ & $3.0(1.5-6.9)$ & n.s. \\
\hline $\mathrm{m}-\mathrm{GGT}^{\mathrm{a}}$ & $2.0(0.7-4.6)$ & $2.1(1.1-6.7)$ & n.s. \\
\hline s-GGT ${ }^{\mathrm{a}}$ & $15.3(7.1-56.7)$ & $30.1(9.2-94.8)$ & n.s. \\
\hline f-GGT & $14.2(8.2-19.0)$ & $14.4(10.9-21.1)$ & n.s. \\
\hline $\mathrm{b} / \mathrm{s}$ ratio ${ }^{\mathrm{a}}$ & $0.17(0.11-0.25)$ & $0.11(0.05-0.25)$ & n.s. \\
\hline
\end{tabular}

Data are reported as median (25th-75th percentile).

a Student's $t$ test has been performed on ln-transformed data.

significantly higher in the latter group $(P<0.01$; Table 2$)$, but in both cases significantly lower than in controls $(P<0.001$ for both comparisons). Within abstainers no differences were found in $b / s$ ratio, between $\mathrm{HCV} / \mathrm{HBV}$ positive or negative subjects (Table 2 ).

\section{Discussion}

In the present study we show that the elevation of total GGT in alcohol addicts is associated with a prominent s-GGT increase, and a lesser increase of all three other fractions. For this reason, the b/s ratio resulted significantly lower than in controls.

This GGT fraction pattern corresponds to that found in subjects with chronic viral hepatitis $\mathrm{C}(\mathrm{CHC})$, but not in subjects with nonalcoholic fatty liver disease (NAFLD) who are characterized by b/s ratio values comparable to those of healthy subjects (Franzini et al., 2011). Interestingly, despite similar absolute values of total GGT and its fractions, the decrease in $\mathrm{b} / \mathrm{s}$ ratio was more marked in alcohol addicts proved to be positive for viral infection, suggesting that $b / s$ ratio is a distinct and potentially quantitative biomarker of hepatocellular damage in alcoholism, even in the presence of viral infection.

Abstinence from alcohol resulted in lowering of total GGT values: b-GGT and f-GGT fractions returned to normal values, while m-GGT and s-GGT levels remained persistently high, in addition to a lower $\mathrm{b} / \mathrm{s}$ ratio than in controls. This finding confirms $\mathrm{b} / \mathrm{s}$ ratio as a sensitive biomarker of persistent liver damage, independently from total GGT level. In addition, the differential decrease in the GGT fractions in alcohol abstainers suggests that GGT fraction analysis might perform better than total GGT, as for monitoring abstinence.

Concerning the interference between viral hepatitis and alcohol addiction, the $\mathrm{b} / \mathrm{s}$ ratio showed a significant difference between $\mathrm{HCV} / \mathrm{HBV}$ positive and negative alcohol addicts, but not among $\mathrm{HCV} / \mathrm{HBV}$ positive and negative abstainers; anyway it is likely to be due to the modest sample size (6 positive vs. 18 negative abstainers); larger studies are needed to fully explore the diagnostic value of the $\mathrm{b} / \mathrm{s}$ ratio in this field.

In a previous study, we showed that fractional GGT analysis showed higher specificity and sensitivity for the direct and differential diagnosis of NAFLD and CHC in comparison to total GGT activity (Franzini et al., 2011). When considering that among NAFLD subjects, high serum GGT levels have been already identified as predictors of liver fibrosis risk (Tahan et al., 2008), further and larger prospective studies are needed to establish the potential value of the individual GGT fractions and of the b/s ratio, either in the screening of alcohol abuse, or as predictors of chronic liver damage in alcohol abusers.

\section{Role of funding source}

This work was supported by Institutional funding (G. Monasterio Foundation CNR-Regione Toscana, Scuola Sant'Anna and University of Pisa, Italy). All funding bodies had no further role in study design; in the collection, analysis and interpretation of data; in the writing of the report; or in the decision to submit the paper for publication.

\section{Contributors}

Authors AP, ME and MM designed the study and wrote the protocol. Author TV, MM and VC selected participants to the study and provided plasma samples. Author IF performed fractional GGT analysis. Author MF undertook the statistical analysis with significant scientific input from ME and AP. Author MF wrote the first draft of the manuscript and authors $\mathrm{AP}, \mathrm{ME}$ and MM contributed to the main content and provided critical comments on the final draft. All authors approved the final manuscript.

\section{Conflict of interest}

No conflict declared.

\section{Acknowledgements}

The authors thank the study participants for their contribution to the research.

\section{References}

Basra, S., Anand, B.S., 2011. Definition, epidemiology and magnitude of alcoholic hepatitis. World J. Hepatol. 3, 108-113.

Bedogni, G., Miglioli, L., Masutti, F., Tiribelli, C., Marchesini, G., Bellentani, S., 2005 Prevalence of and risk factors for nonalcoholic fatty liver disease: the Dionysos nutrition and liver study. Hepatology 42, 44-52.

Das, S.K., Dhanya, L., Vasudevan, D.M., 2008. Biomarkers of alcoholism: an updated review. Scand. J. Clin. Lab. Invest. 68, 81-92.

Franzini, M., Bramanti, E., Ottaviano, V., Ghiri, E., Scatena, F., Barsacchi, R., Pompella, A., Donato, L., Emdin, M., Paolicchi, A., 2008a. A high performance gel filtration chromatography method for gamma-glutamyltransferase fraction analysis. Anal. Biochem. 374, 1-6.

Franzini, M., Fornaciari, I., Fierabracci, V., Elawadi, H.A., Bolognesi, V., Maltinti, S., Ricchiuti, A., De Bortoli, N., Marchi, S., Pompella, A., Passino, C., Emdin, M., Paolicchi, A., 2011. Accuracy of b-GGT fraction for the diagnosis of non-alcoholic fatty liver disease. Liver Int. 32, 629-634.

Franzini, M., Ottaviano, V., Fierabracci, V., Bramanti, E., Zyw, L., Barsacchi, R., Scatena, F., Boni, C., Mammini, C., Passino, C., Pompella, A., Emdin, M., Paolicchi, A., 2008b. Fractions of plasma gamma-glutamyltransferase in healthy individuals: reference values. Clin. Chim. Acta 395, 188-189. 
Huseby, N.E., 1982. Multiple forms of serum gamma-glutamyltransferase. Association of the enzyme with lipoproteins. Clin. Chim. Acta 124, 103-112.

Nemesanszky, E., Lott, J.A., 1985. Gamma-glutamyltransferase and its isoenzymes: progress and problems. Clin. Chem. 31, 797-803.

Rosalki, S.B., Rau, D., 1972. Serum-glutamyl transpeptidase activity in alcoholism. Clin. Chim. Acta 39, 41-47.
Schellenberg, F., Schwan, R., Mennetrey, L., Loiseaux, M.N., Pagès, J.C., Reynaud, M. 2005. Dose-effect relation between daily ethanol intake in the range 0-70 grams and \%CDT value: validation of a cut-off value. Alcohol Alcohol. 40, 531-534.

Tahan, V., Canbakan, B., Balci, H., Dane, F., Akin, H., Can, G., Hatemi, I., Olgac, V., Sonsuz, A., Ozbay, G., Yurdakul, I., Senturk, H., 2008. Serum gammaglutamyltranspeptidase distinguishes non-alcoholic fatty liver disease at high risk. Hepatogastroenterology 55, 1433-1438. 